\title{
PROJETO E CONSTRUÇÃO DE UM SEDIMENTADOR EM ESCALA DE LABORATÓRIO
}

\author{
A. B. L. SPIRANDELI ${ }^{1}$, A. S. SOUZA ${ }^{1 *}$, C. F. PINTO ${ }^{1}$, L. MARTINELLI ${ }^{1}$, M. E. SPERETTA ${ }^{1}$, \\ K. G. SANTOS ${ }^{1}$ \\ ${ }^{1}$ Universidade Federal do Triângulo Mineiro, Departamento de Engenharia Química \\ *e-mail: amarilis.sev@gmail.com
}

\begin{abstract}
RESUMO
A sedimentação é um processo no qual um sólido suspenso em um líquido é separado devido à diferença entre as densidades e concentrações das fases presentes na suspensão, por ação do campo gravitacional. Os métodos existentes para dimensionamento de sedimentadores são empregados para equipamentos de alta capacidade, de uso industrial ou em estações de tratamento de água e esgoto. Neste trabalho, foi projetado e construído um sedimentador em escala de laboratório (piloto) pelos métodos de Biscaia Jr e Kynch. $\mathrm{O}$ sedimentador contínuo foi projetado e construído de acordo com as dimensões fornecidas pelo método citado de Biscaia Jr., pelo qual uma concentração de alimentação de suspensão de cal hidratada de $70 \mathrm{~g} \mathrm{~L}^{-1}$ foi selecionada, com tanque sedimentador de $52 \mathrm{~cm}$ de diâmetro e altura total de $31 \mathrm{~cm}$. A concentração do lodo obtida experimentalmente foi superior ao predito pelo método de Biscaia Jr., resultando em espessado de $425 \mathrm{~g} \mathrm{~L}^{-1}$. O líquido clarificado obtido apresentou baixas turbidez e concentração de sólidos. A eficiência de separação sólido-líquido obtida foi de 95,7\%. Assim, considerou-se o equipamento construído com ótima eficiência de separação.
\end{abstract}

\section{INTRODUÇÃO}

A sedimentação é uma operação de separação sólido-líquido baseada na diferença entre as concentrações das fases presentes na suspensão a ser processada, sujeitas à ação do campo gravitacional (CREMASCO, 2012).

Os equipamentos sedimentadores são largamente utilizados, por exemplo, na indústria de mineração para a obtenção de polpas com concentrações adequadas a um determinado processo subsequente, espessamento de rejeitos com concentração elevada de partículas, recuperação de água para reciclo industrial, recuperação de partículas ou solução de operações de lixiviação, dentre outros. (FRANÇA E MASSARANI, 2004). $\begin{array}{cccc}\text { Os } & \text { sedimentadores } & \text { podem } & \text { ser } \\ \text { classificados } & \text { em duas categorias: } & \text { os }\end{array}$ clarificadores, quando o interesse é a obtenção de um sobrenadante limpo, ou seja, o objetivo do processo é a remoção de uma quantidade relativamente pequena de partículas de um efluente; e os espessadores, quando o principal interesse é a obtenção de uma suspensão mais concentrada. Os espessadores são os mais utilizados nas indústrias, já que, na maioria das vezes, o produto de interesse é a lama ou lodo (maior concentração de sólidos).

De acordo com CREMASCO (2012), pode-se ter uma ideia qualitativa da operação de sedimentação analisando a velocidade terminal da partícula isolada. Partículas esféricas, maiores em diâmetro e massa específica apresentarão maior valor para a velocidade terminal. Assim, decantarão mais 
rapidamente se comparadas a partículas não esféricas e de diâmetro e massa específica menores. Ademais, fatores como a natureza morfológica e granulométrica das partículas, além de características do tanque de sedimentação (altura, diâmetro, vazão no overflow e no underflow) podem afetar essa operação unitária.

Os sedimentadores industriais operam continuamente e geralmente são construídos com seção circular, dotada de uma parte cilíndrica e uma cônica que tem como finalidade facilitar a retirada do lodo ou lama do equipamento. $\mathrm{Na}$ parte superior do equipamento há um vertedouro, por onde transborda a corrente de líquido clarificado. A alimentação da suspensão que se deseja processar é posicionada na região central do equipamento e pode ser feita no topo ou no interior do mesmo (AZEVEDO, 2009). Para executar uma separação sólido-líquido, o projeto do sedimentador contínuo está basicamente relacionado ao cálculo da área de seção de sedimentação e da altura do equipamento.

Observou-se que existem dificuldades e incertezas no estabelecimento das equações constitutivas para as lamas compressíveis, o que levou o projeto do sedimentador a ser baseado nos ensaios em batelada ou testes de proveta conduzidos diretamente com a suspensão a ser tratada (MASSARANI, 2001).

Os métodos existentes para dimensionamento de sedimentadores têm sua aplicação voltada para equipamentos de alta capacidade, de uso industrial ou nas estações de tratamento de água (ETA) ou esgoto (ETE). Para estudos dessa operação unitária em laboratório, necessita-se equipamentos de menor escala e, de preferência, baixo custo, que possam ter sua eficiência e qualidade do produto final verificada em relação à partícula utilizada e aos valores de projeto. Com essa motivação, foi feita a aplicação dos métodos de Kynch (1952) e Biscaia Jr. (1982) na projeção e construção de um sedimentador em escala de laboratório (piloto), sendo sua eficiência verificada por meio da determinação da concentração de sólidos no líquido clarificado e no lodo do sedimentador, sendo comparados esses resultados com os valores do projeto.

\section{METODOLOGIA}

\subsection{Cálculo da Concentração do Lodo pelos Métodos de Biscaia Jr. e Kynch}

Foram feitos testes de proveta para suspensões de cal hidratada (Itaú ${ }^{\circledR}$ ) nas concentrações de 30,50 e $70 \mathrm{~g} \mathrm{~L}^{-1}$. Provetas de $100 \mathrm{~mL}$ foram completadas com as suspensões e agitadas com bastão de vidro. Ao final da agitação, um cronômetro foi acionado e registou-se a variação da altura de líquido clarificado com o tempo em cada proveta.

Para o método de Biscaia Jr., os diâmetros foram calculados para uma vazão de alimentação no sedimentador de $25 \mathrm{~cm}^{3} \mathrm{~s}^{-1}$, partindo da Equação 1.

$z_{\min }=\frac{C_{a} z_{0}}{C_{L}}$

Com o valor de zmin, pode-se ler o valor $\theta_{\text {min }}$ correspondente no gráfico do teste de proveta e a área da base do sedimentador $(A)$ pode ser calculada pela Equação 2. Com o seu valor, é possível fazer a determinação do diâmetro.

$A=\frac{F}{z_{0} \theta_{\min }}$

Também foi determinada a altura da região de concentração variável $\left(H_{C}\right)$ e do fundo ou parte cônica do equipamento $(H 2)$. $H_{C}$ é calculado pela Equação 3 (FRANÇA E MASSARANI, 2004) e $H 2$ é dado pela Equação 4.

$$
H_{C}=\frac{4}{3} \frac{F C_{a} \tau}{A p_{s}}\left(\frac{\rho_{s}-\rho}{\rho_{L}-\rho}\right)
$$


$H 2=0,073 \cdot D$

As densidades da cal hidratada e da água à temperatura ambiente foram de $2,21 \mathrm{~g}$ $\mathrm{cm}^{-3}$ e $0,998 \mathrm{~g} \mathrm{~cm}^{-3}$, respectivamente.

O dimensionamento do sedimentador contínuo foi realizado considerando as três concentrações iniciais de suspensão, a partir das quais foram calculados os diâmetros do sedimentador em cada condição operacional. A partir dos resultados obtidos no dimensionamento, optou-se pela construção do sedimentador capaz de tratar a maior concentração inicial de suspensão estudada.

$\mathrm{O}$ método de Kynch foi aplicado para cálculo do diâmetro do sedimentador para a concentração de alimentação de $70 \mathrm{~g} \mathrm{~L}^{-1}$, a fim de comparar seu resultado com o do método anterior. A partir da obtenção dos valores de $v$ e $z i$ na curva de pontos experimentais, foi calculado o máximo diâmetro obtido pela Equação 5. $\mathrm{O}$ valor $z i$ representa a altura que a suspensão ocuparia se todas as regiões tivessem a concentração $C$ (Equação 6), dado pelo valor $z$ no qual a reta que tangencia a curva dos pontos experimentais intercepta $\mathrm{o}$ eixo $\mathrm{y} ; \quad v$ corresponde à velocidade de sedimentação, que é o valor da inclinação dessa reta.

$$
\begin{aligned}
& \frac{F}{A}=\frac{1}{C_{a}} \frac{1}{\left(\frac{1}{C}-\frac{1}{C_{L}}\right)} \\
& C=\frac{C_{a} z_{0}}{z i}
\end{aligned}
$$

\subsection{Construção da Unidade Experimental}

A Figura 1 apresenta o sistema de sedimentação construído, com seus respectivos componentes indicados.

\subsubsection{Sedimentador}

Foi utilizado um reservatório plástico de $31 \mathrm{~cm}$ de altura e de seção trapezoidal com diâmetros menor e maior iguais a 52 e $59 \mathrm{~cm}$, respectivamente.

Um conduíte foi cortado longitudinalmente e colado ao longo da borda do reservatório como vertedouro para coletar o líquido clarificado. O lodo era recolhido por um tubo de silicone de 1 in de diâmetro conectado no fundo do sedimentador. Uma válvula regulava a vazão de saída do lodo.

O raspador (Figura 2) foi projetado com 11 pás de $5 \mathrm{~cm}$ formando um ângulo de $53^{\circ}$ com seu suporte, igualmente espaçadas. A base das pás possuía uma folha de EVA para suavizar o seu movimento. Um motor fornecia uma rotação de $6 \mathrm{rpm}$.

\subsubsection{Alimentação}

A alimentação era feita por diferença de altura, utilizando dois reservatórios plásticos.

O reservatório auxiliar possuía $10 \mathrm{~L}$ de capacidade e mantinha a suspensão agitada por meio de um misturador de propulsão elétrico. Este, por sua vez, foi o responsável por fornecer a suspensão ao reservatório de alimentação a fim de manter o seu nível aproximadamente constante para controlar a vazão de alimentação do sedimentador. Quando necessário, foi acionado um dispositivo de reabastecimento do seu volume, operado manualmente com a suspensão. $\mathrm{O}$ reservatório de alimentação continha $30 \mathrm{~L}$ de suspensão mantidos constantes por uma válvula de boia (Amanco®) conectada ao reservatório auxiliar.

Uma bomba centrífuga realizava a agitação da suspensão nesse reservatório. A alimentação era feita no centro do sedimentador, com um tubo mergulhado $20 \mathrm{~cm}$ no tanque e possuía sua vazão controlada por uma válvula. 
Figura 1 - Esquema do sistema construído: (1) - Reservatório auxiliar; (2) - Reservatório de alimentação; (3)

- Sedimentador; (4) - Tanque de coleta de clarificado; (5) - Tanque de coleta de lodo.



Fonte: Autor (2015).

Figura 2 - Raspador do sedimentador.

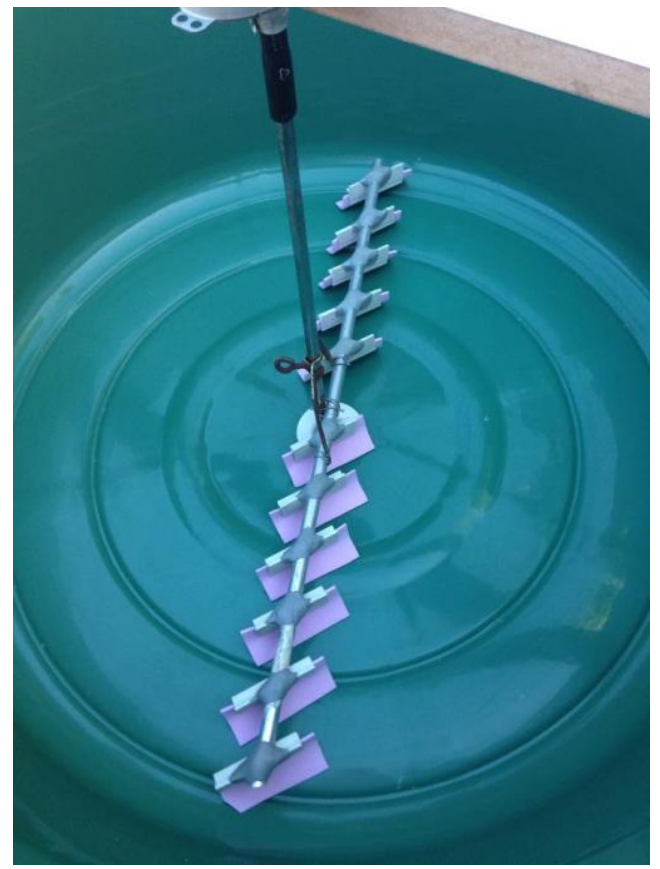

\subsection{Operação e análise}

O início da operação do sedimentador foi feita completando o reservatório com a suspensão de cal hidratada na mesma concentração da alimentação $\left(70 \mathrm{~g} \mathrm{~L}^{-1}\right)$, como num processo batelada.

Após alguns minutos, a válvula do tanque de alimentação foi aberta fornecendo vazão de suspensão de aproximadamente 25 $\mathrm{cm}^{3} \mathrm{~s}^{-1}$

Em seguida, foi aberta a válvula de saída de lodo. Quando o clarificado começou a transbordar, foi feita a primeira coleta de clarificado e lodo. Após 90 minutos de operação, foi realizada uma nova coleta de amostras de clarificado e do lodo.

As amostras foram pesadas antes e após serem levadas para a estufa à $100{ }^{\circ} \mathrm{C}$ e $24 \mathrm{~h}$, a

Fonte: Autor (2015). 
fim de determinar a concentração de sólidos presentes por litro de suspensão.

As vazões do underflow (espessado) e do overflow (líquido clarificado) foram calculadas por meio dos balanços de massa global e para as partículas, representados pelas Equações 7 e 8.

$F_{m}=F_{\text {under }}+F_{\text {over }}$

$F_{m} C_{a}=F_{\text {under }} \cdot C_{\text {under }}+F_{\text {over }} \cdot C_{\text {over }}$

Em que $F_{m}, F_{\text {under }}, F_{\text {over }}$ são as vazões mássicas de alimentação, espessado e clarificado, respectivamente; e $C_{a}, C_{\text {under }} \mathrm{e}$ $C_{\text {over }}$ são as concentrações mássicas das correntes de alimentação, espessado e clarificado, respectivamente.

\subsection{Fluidodinâmica Computacional do Sedimentador}

A fim de verificar o efeito da posição da alimentação dentro do sedimentador, e como seria o perfil de velocidades do fluido dentro deste, foi realizada uma simulação empregando a fluidodinâmica computacional.

Foram definidas: a velocidade inicial do fluido, a geometria do equipamento e as propriedades do fluido (densidade, temperatura, viscosidade).

Para tal, a malha computacional correspondente à geometria do sedimentador foi confeccionada, em $2 \mathrm{D}$, empregando $\mathrm{o}$ software GAMBIT, que disponibiliza uma interface com o "software" de fluidodinâmica computacional FLUENT ${ }^{\circledR}$. A malha computacional usada é estruturada, com 9930 elementos quadrangulares.

As condições de contorno foram adequadamente definidas: nas paredes, a velocidade do fluido é nula; na face de entrada é possível inserir a velocidade do fluido; nas faces de saída, define-se a pressão como sendo atmosférica.

O campo de velocidades do fluido é obtido pela resolução da equação da continuidade e da equação de quantidade de movimento para a fase fluida, descritas pelas Equações 9 e 10.

$\frac{\partial}{\partial t}(\rho)+\nabla(\rho \vec{v})=S_{m}$

$\frac{\partial}{\partial t}(\rho \vec{v})+\nabla \cdot(\rho \vec{v} \vec{v})=-\nabla p+\nabla \cdot \overline{\bar{\tau}}+\rho \vec{g}+\vec{F}$

Em que $S_{m}$ representa a massa adicionada para a fase contínua (fluida) devido à dispersão da fase discreta (partículas); - $\nabla p$ é gradiente de pressão estática; $\rho \vec{g}$ representa a força gravitacional e $\vec{F}$ as forças de corpo, $\overline{\bar{\tau}}$ é tensor tensão de deformação da fase fluida, que pode ser descrito por:

$\overline{\bar{\tau}}=\mu\left[\left(\nabla \vec{v}+\vec{v}^{T}\right)-\frac{2}{3} \nabla \cdot \vec{v} I\right]$

Em que $\mu$ é a viscosidade do fluido; $\vec{v}$ é o tensor unitário e o termo $2 / 3 \nabla \cdot \vec{v} I$ está relacionado ao efeito da dilação de volume.

$\mathrm{O}$ sistema de equações foi então resolvido pelo método de volumes finitos, para obtenção dos perfis em estado estacionário.

Foi adotado um critério de convergência de $10^{-6}$ para a equação da continuidade e de $10^{-}$ 4 para as equações de quantidade de movimento do fluido.

O acoplamento entre velocidade e pressão foi feito pelo esquema SIMPLE. Para a discretização espacial foi utilizado o método upwind de segunda ordem, para as equações de momento.

Esta simulação, apesar de não conter as partículas, pode mostrar como é a trajetória do fluido dentro do equipamento.

\section{RESULTADOS E DISCUSSÕES}

\subsection{Dimensionamento do equipamento}

Os gráficos obtidos pelos testes de proveta estão dispostos na Figura 3. 
A predominância de uma tendência linear nos pontos experimentais manteve-se por mais tempo nas maiores concentrações. $\mathrm{O}$ tempo de compactação também foi mais longo para as maiores concentrações, visto que sua altura de compactação é maior devido à quantidade de partículas.

A Tabela 1 apresenta as dimensões obtidas pelo método de Biscaia Jr. para cada teste de proveta feito, considerando uma $C_{L}$ de $300 \mathrm{~g} \mathrm{~L}^{-1}$. Foi adicionado $10 \%$ do valor calculado como margem de segurança.

Tabela 1 - Diâmetros correspondentes às concentrações de alimentação para $F=25 \mathrm{~cm}^{3} \mathrm{~s}^{-1}$

\begin{tabular}{cc}
\hline$C_{a}(\mathrm{~g} \mathrm{~L})$ & Diâmetro $(\mathrm{cm})$ \\
\hline 30 & 25 \\
50 & 35 \\
70 & 51 \\
\hline
\end{tabular}

Fonte: Autor (2015).

Figura 3 - Pontos experimentais dos testes de proveta

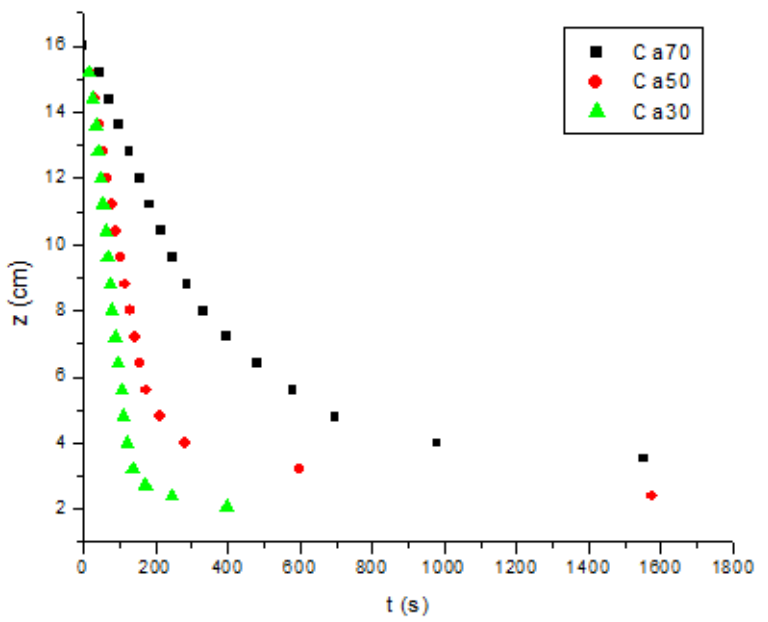

Fonte: Autor (2015).

A concentração de $70 \mathrm{~g} \mathrm{~L}^{-1}$, com zmín de $3,7 \mathrm{~cm}$, foi escolhida para a construção do sedimentador, visto que foi aquela que apresentou um diâmetro que condizia com o material disponível para a construção.

A Figura 4 apresenta o gráfico gerado como resultado do teste de proveta para a concentração de alimentação escolhida. Foi feito um ajuste não linear da distribuição de pontos experimentais, obtendo um coeficiente de correlação de 0,995 e Chi-Quadrado de 0,0818. A função obtida pelo ajuste está indicada na Equação 12:

$z=3,16+13,5 \cdot \exp (-t / 342)$

Com a derivada da Equação 12 determinou-se os valores de $v$ e $z i$ correspondentes a cada ponto experimental da curva da Figura 4. O diâmetro calculado pelo método de Kynch para $C_{a}$ de $70 \mathrm{~g} \mathrm{~L}^{-1}$, adicionados $10 \%$ de margem de segurança, também foi de $51 \mathrm{~cm}$, o mesmo valor obtido pelo método anterior.

O ajuste foi utilizado para auxiliar no cálculo do valor de $\tau$, na determinação de $H c$. Com a derivada da Equação 12 foi possível determinar o ponto correspondente à $C_{L}$, que é o tempo no qual a reta que intercepta o gráfico em Zmin tangencia a curva ajustada, que equivale a 1693 s. Subtraindo esse tempo ao que corresponde ao final da tendência linear, obtém-se um valor de $\tau$ de 1406 s. Foi calculado um $\rho_{L}$ de $1,16 \mathrm{~g} \mathrm{~cm}^{-3}$.

Figura 4 - Ajuste não linear dos dados experimentais do teste de proveta para $C_{a}=70 \mathrm{~g} \mathrm{~L}$

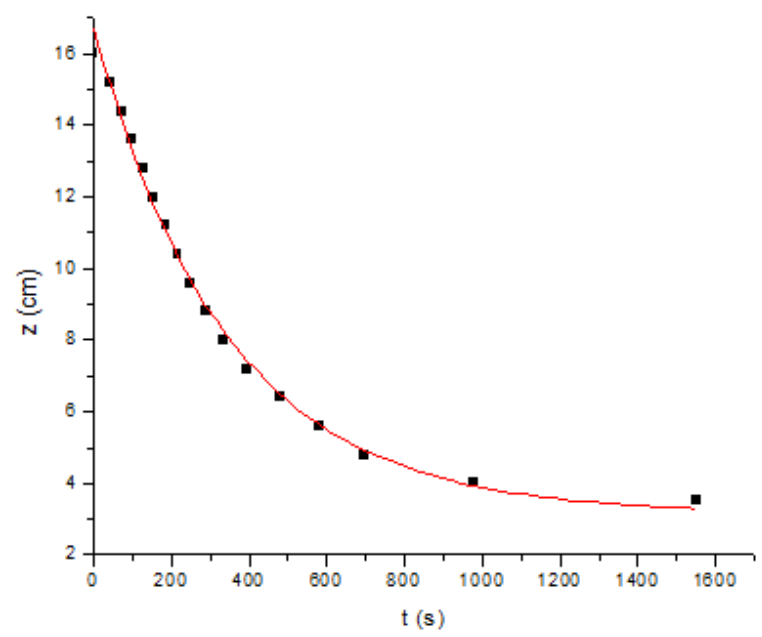

Fonte: Autor (2015). 
Os valores obtidos para $\mathrm{Hc}$ e $\mathrm{H} 2$ foram de $6,4 \mathrm{~cm}$ e $3,4 \mathrm{~cm}$, respectivamente. Para o cálculo de $H 1$ foi desconsiderado o valor de projeto (entre 0,45 e 0,75 ), já que a aplicação deste trabalho é um sedimentador de escala reduzida. Portanto, foi atribuído o valor de 20 cm para $H 1$.

Como o recipiente disponível para a construção não apresentava a parte cônica, esta foi desprezada na construção do equipamento.

\subsection{Operação do Sedimentador Contínuo}

A Tabela 2 apresenta as concentrações em gramas de sólido por litro de suspensão das amostras coletadas no início e após 90 minutos de operação, comparadas com os valores de projeto. No início da operação, foi considerado que a concentração de sólidos da alimentação encontrava-se conforme os valores de projeto.

A partir dos balanços de massa global e para o sólido particulado, representados pelas Equações 5 e 6, foi possível obter os valores das vazões volumétricas das correntes de clarificado e lodo, que resultaram nos valores de 20,96 e 4,04 $\mathrm{cm}^{3} \mathrm{~s}^{-1}$, respectivamente.

Também foram calculadas as densidades das correntes de lodo e clarificado. A corrente de clarificado apresentou densidade muito próxima à do fluido, visto que há uma fração mínima de partículas na suspensão. Já o lodo, apresentou densidade maior que a prevista pelo Método de Biscaia, sendo esta de $1,221 \mathrm{~g} \mathrm{~cm}^{-3}$.

Tabela 2 - Concentração das amostras coletadas

\begin{tabular}{ccccccc}
\hline \multirow{2}{*}{ Suspensão } & \multicolumn{3}{c}{ Concentração $\left(\mathrm{g} \mathrm{L}^{-1}\right)$} & & $\begin{array}{c}\text { Vazão no final } \\
\left(\mathrm{cm}^{3} \mathrm{~s}^{-1}\right)\end{array}$ & $\begin{array}{c}\text { Densidade da } \\
\text { Suspensão } \\
\left.\mathrm{nyynnn}^{-3}\right)\end{array}$ \\
\hline Início & Final & Biscaia $\mathrm{Jr} / \mathrm{Kynch}$ & & 20,96 & 0,999 \\
Clarificado & 2,4 & 2,3 & - & 25,00 & 1,035 \\
Alimentação & 70,0 & 70,0 & 70 & 4,04 & 1,221 \\
Lodo & 224,3 & 425,1 & 300 & & \\
\hline
\end{tabular}

Fonte: Autor (2015).

Durante a coleta, observou-se a deposição de cal nas paredes do tubo, o que provocou uma vazão oscilante e um aumento na compactação e concentração do lodo na saída.

Foi observado também que a agitação dos reservatórios de alimentação pode não ter sido suficiente para a quantidade de cal utilizada, sendo necessária uma agitação mais intensa nos tanques de alimentação.

Ao longo de toda a operação, o líquido clarificado apresentou baixa turbidez. Houve baixa variação da concentração de sólidos coletados no início e após os 90 minutos de operação do sistema. Comparando o valor da concentração da amostra de alimentação e o do clarificado no final, pode-se calcular uma eficiência de remoção de cal da água de aproximadamente $95,7 \%$.
É válido acrescentar que a cal possui uma solubilidade em água de $1,65 \mathrm{~g} \mathrm{~L}^{-1}$ à temperatura de $20{ }^{\circ} \mathrm{C}$ (GREEN; PERRY, 1999), resultando em elevada cor aparente do clarificado, que não pode ser removida por decantação. Uma parcela das partículas presentes no clarificado constituíam-se de sólidos flutuantes e impurezas presentes na cal.

A Figura 4 apresenta o contorno de velocidade axial do fluido dentro do recipiente com a mesma geometria do sedimentador. Observa-se que há uma recirculação de fluido próxima à alimentação, mas que as maiores velocidades de fluido são apenas próximo à alimentação e à saída da corrente de lodo. $\mathrm{Na}$ região superior de saída de clarificado, a velocidade do fluido é baixa, e portanto, a probabilidade de arraste das partículas é pequena. Assim, acredita-se que a alimentação 
da suspensão no sedimentador não está causando a ressuspensão dos sólidos, o que confere com a elevada eficiência de separação do equipamento proposto.

Figura 5 - Contorno de velocidade axial do fluido $(\mathrm{m} / \mathrm{s})$.
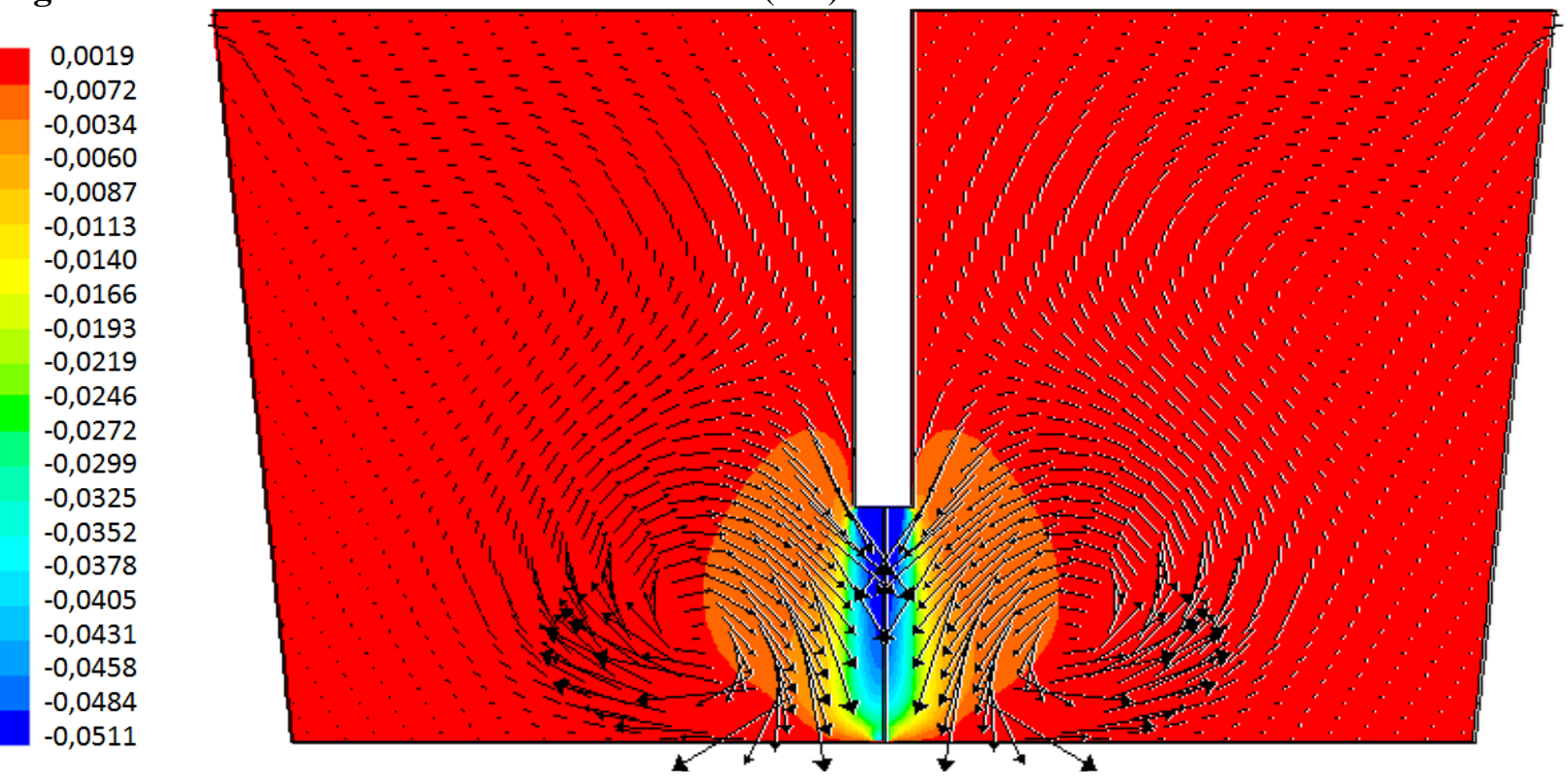

Fonte: Autor (2015).

\section{CONCLUSÃO}

O sedimentador contínuo em escala de laboratório foi construído conforme os valores de projeto calculados pelo método de Biscaia Jr., que forneceram um diâmetro de $51 \mathrm{~cm} \mathrm{e}$ uma altura total de $30 \mathrm{~cm}$ para uma vazão de alimentação de $25 \mathrm{~cm}^{3} \mathrm{~s}^{-1}$ e concentração de 70 g $\mathrm{L}^{-1}$ de suspensão de cal, prevendo uma concentração de lodo de $300 \mathrm{~g} \mathrm{~L}^{-1}$. O método de Kynch resultou no mesmo diâmetro calculado por Biscaia Jr.

Por meio da simulação feita empregando fluidodinâmica computacional foi analisado o contorno de velocidade axial do fluido dentro do equipamento, mostrando que não houve ressuspensão dos sólidos devido à posição do tubo de alimentação.

O resultado das análises feitas durante a operação do sedimentador remeteram numa produção de clarificado com baixas turbidez e concentração de sólidos, implicando numa eficiência de separação de partículas de 95,7\%.

Para melhorias da condução deste projeto e recomendação para futuros, é necessária uma maior agitação dos tanques de alimentação e a utilização de uma bomba de parafusos para retirada de lodo, evitando deposição de sólidos.

\section{NOMENCLATURA}

A Área da base do tanque sedimentador, $\mathrm{M}^{0} \mathrm{~L}^{2} \mathrm{~T}^{0}$

$C_{a}$ Concentração da suspensão de alimentação, $\mathrm{M}^{1} \mathrm{~L}^{-3} \mathrm{~T}^{0}$

$C_{L} \quad$ Concentração do lodo, $\mathrm{M}^{1} \mathrm{~L}^{-3} \mathrm{~T}^{0}$

$C_{\text {over }}$ Concentração mássica da corrente de líquido clarificado, $\mathrm{M}^{1} \mathrm{~L}^{-3} \mathrm{~T}^{0}$

$C_{\text {under }}$ Concentração mássica da corrente de espessado, $\mathrm{M}^{1} \mathrm{~L}^{-3} \mathrm{~T}^{0}$

$F \quad$ Vazão de alimentação, $\mathrm{M}^{0} \mathrm{~L}^{3} \mathrm{~T}^{-1}$

$F_{m} \quad$ Vazão mássica de alimentação, $\mathrm{M}^{1} \mathrm{~L}^{0} \mathrm{~T}^{-1}$ 
$F_{\text {over }}$ Vazão mássica de clarificado, $\mathrm{M}^{1} \mathrm{~L}^{0} \mathrm{~T}^{-1}$

$F_{\text {under }}$ Vazão mássica de espessado, $\mathrm{M}^{1} \mathrm{~L}^{0} \mathrm{~T}^{-1}$

$\vec{F} \quad$ Forças de corpo, $\mathrm{M}^{1} \mathrm{~L}^{1} \mathrm{~T}^{-2}$

$g \quad$ Aceleração da gravidade, $\mathrm{M}^{0} \mathrm{~L}^{1} \mathrm{~T}^{-2}$

H1 Altura da região de líquido clarificado, $\mathrm{M}^{0} \mathrm{~L}^{1} \mathrm{~T}^{0}$

Hc Altura da região de concentração variável, $\mathrm{M}^{0} \mathrm{~L}^{1} \mathrm{~T}^{0}$

H2 Altura do fundo ou parte cônica do equipamento, $\mathrm{M}^{0} \mathrm{~L}^{1} \mathrm{~T}^{0}$

$S_{m}$ Massa adicionada para a fase contínua, $\mathrm{M}^{1} \mathrm{~L}^{0} \mathrm{~T}^{0}$

$t \quad$ Tempo, $\mathrm{M}^{0} \mathrm{~L}^{0} \mathrm{~T}^{1}$

$v \quad$ Velocidade de sedimentação, $\mathrm{M}^{0} \mathrm{~L}^{1} \mathrm{~T}^{-1}$

$z \quad$ Altura da interface, $\mathrm{M}^{0} \mathrm{~L}^{1} \mathrm{~T}^{0}$

$z i \quad$ Altura ocupada pela suspensão para uma concentração $C$ em todas as regiões, $\mathrm{M}^{0} \mathrm{~L}^{1} \mathrm{~T}^{0}$

zmin Valor associado a $\theta_{\text {mín }}$ pelo gráfico resultante do teste de proveta, $\mathrm{M}^{0} \mathrm{~L}^{1} \mathrm{~T}^{0}$

zo Altura máxima da suspensão na proveta, $\mathrm{M}^{0} \mathrm{~L}^{1} \mathrm{~T}^{0}$

$\nabla p \quad$ Gradiente de pressão estática, $\mathrm{M}^{1} \mathrm{~L}^{-1} \mathrm{~T}^{-2}$

$\theta_{\text {min }}$ Tempo associado a $z_{\text {min }}, \mathrm{M}^{0} \mathrm{~L}^{0} \mathrm{~T}^{1}$

$\mu \quad$ Viscosidade do fluido, $\mathrm{M}^{1} \mathrm{~L}^{-1} \mathrm{~T}^{-1}$

$\rho \quad$ Densidade da água, $\mathrm{M}^{1} \mathrm{~L}^{-3} \mathrm{~T}^{0}$

$\rho_{s} \quad$ Densidade da cal hidratada, $\mathrm{M}^{1} \mathrm{~L}^{-3} \mathrm{~T}^{0}$

$\rho_{L} \quad$ Densidade do lodo, $\mathrm{M}^{1} \mathrm{~L}^{-3} \mathrm{~T}^{0}$

$\overline{\bar{\tau}}$ Tensor tensão de deformação da fase fluida

\section{REFERÊNCIAS}

AZEVEDO, C. G. Simulação da operação de sedimentadores contínuos. 2009. 229f. Dissertação (Pós-graduação em Engenharia) Faculdade de Engenharia Química, Universidade Federal de Uberlândia, Uberlândia, 2009.

CREMASCO, M. A. Operações unitárias em sistemas particulados e fluidomecânicos. São Paulo: Bluthcer, 2012. 423p.
FRANÇA, S. C.; MASSARANI, G. Separação sólido-líquido. Comunicação Técnica - CETEM. Rio de Janeiro, 2004.

GREEN, D. PERRY, R. Perry's Chemical Engineers' Handbook. 7. ed. Nova York: McGrall-Hill, 1999.

MASSARANI, G. Fluidodinâmica em sistemas particulados. 2. ed. Rio de Janeiro: Ed. UFRJ, 2001. 